\title{
Analysis of High Efficiency Electromagnetically Induced Transparency in Potassium Vapor
}

\author{
S. Gozzini ${ }^{a}$, D. Slavov ${ }^{b, *}$, S. Cartaleva ${ }^{b}$, L. Marmugi $^{a}$ And A. Lucchesini $^{a}$ \\ ${ }^{a}$ IPCF-CNR, Area della Ricerca, via Moruzzi 1, 56124 Pisa, Italy \\ ${ }^{b}$ Institute of Electronics, BAS, boul. Tzarigradsko shosse 72, 1784 Sofia, Bulgaria
}

\begin{abstract}
We present a rate-equation theoretical model describing the optical pumping processes on the $\mathrm{K} D_{1}$ line and we then discuss their influence on the electromagnetically induced transparency resonance parameters. We present also a comparison with the results of an experiment performed in cells containing pure alkali metal or added with a few torrs of buffer gas. The model shows that, in the last case, the complete Maxwellisation of the atomic population velocity distribution, along with the overlapping Doppler profiles of the transitions from the ground-states typical of $\mathrm{K}$, leads to a partial compensation of optical pumping and a significant increase of the amplitude of the electromagnetically induced transparency resonances.
\end{abstract}

PACS numbers: 33.57. $+\mathrm{c}, 31.15 . \mathrm{B}, 32.80 . \mathrm{Xx}, 32.50 .+\mathrm{d}, 32.60 .+\mathrm{i}$

\section{Introduction}

Electromagnetically induced transparency (EIT) resonances have been studied mainly in $\mathrm{Rb}$ and Cs vapors, due to the availability of conventional diode lasers matching their resonance lines. Recently strong enhancement of EIT resonance contrast and amplitude has been demonstrated experimentally for EIT prepared in the so-called Hanle configuration with $\mathrm{K}$ atoms contained in antirelaxation coated and buffered cells [1]. Such relevant improvement is a consequence of the reduced optical pumping (OP) in $\mathrm{K}$ due to the significant overlapping of the hyperfine-level Doppler profiles, which does not occur in the case of $\mathrm{Rb}$ and $\mathrm{Cs}$ vapors. Thanks to the velocity changing collisions of $\mathrm{K}$ with buffer gas atoms an efficient repumping is realized: this produces the excitation of both ground levels. We reported in Ref. [2]. that EIT resonances in $\mathrm{K}$ created with $\mathrm{kHz}$ intensity modulated laser light show high contrast and good width parameters. A similar modulation approach has been used for coherent resonance study in $\mathrm{Rb}$, registered as non-linear magneto-optical rotation of the light polarization [3].

\section{Theoretical model}

The theoretical model used here is based on the ones reported in [4] and [5] adapted for the particular case of potassium. We consider a three-level scheme (Fig. 1) with levels $|1\rangle$ and $|2\rangle$ representing the two ground-state hyperfine levels $\left(4^{2} S_{1 / 2} \quad F_{g}=1\right.$ and 2$)$ and level $|3\rangle$ the excited states of $\mathrm{K} D_{1}$-line $\left(4^{2} P_{1 / 2}\right)$. Here $\gamma$ is the excited level spontaneous decay rate, $\Delta_{\mathrm{hf}}=462 \mathrm{MHz}$ is the hyperfine splitting of the ground states and $\omega_{1}$ and $\omega_{2}$ are the optical transition frequencies.

\footnotetext{
* corresponding author; e-mail: slavov_d_g@yahoo.com
}

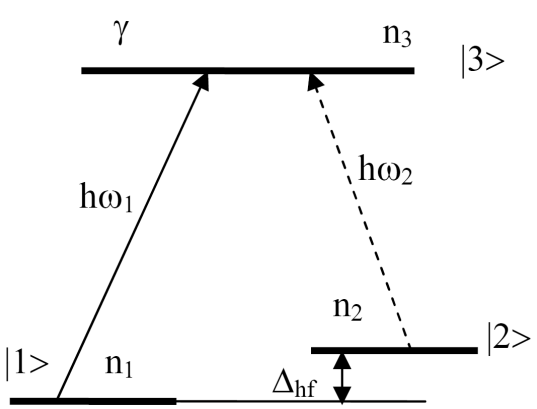

Fig. 1. The energy level diagram used in the theoretical model.

\subsection{The system of differential rate equations}

The system of differential rate equations is the following:

$$
\left\{\begin{array}{l}
\dot{n}_{1}=\left(n_{3}-n_{1}\right) \sigma_{1}+n_{3} \frac{\gamma}{2}+\left(n^{0}-n_{1}\right) \Gamma, \\
\dot{n}_{2}=\left(n_{3}-n_{2}\right) \sigma_{2}+n_{3} \frac{\gamma}{2}+\left(n^{0}-n_{2}\right) \Gamma, \\
\dot{n}_{3}=-\left(n_{3}-n_{1}\right) \sigma_{1}-\left(n_{3}-n_{2}\right) \sigma_{2} \\
\quad-2\left(n_{3} \frac{\gamma}{2}\right)-n_{3} \Gamma,
\end{array}\right.
$$

where number conservation requires

$$
n_{1}+n_{2}+n_{3}=2 n^{0},
$$

$n_{1}(v), n_{2}(v)$ and $n_{3}(v)$ are the population densities of the corresponding levels for atoms within a velocity group between $v$ and $v+\mathrm{d} v$. Here $v$ is the velocity component of the atoms along the laser propagation direction $z$. The cross-section of the optical transitions is expressed as

$$
\sigma_{j}=\left(\frac{I}{\hbar \omega}\right) \sigma_{0} L_{j}(v)=\left(\frac{\gamma I}{2 I_{\mathrm{S}}}\right) L_{j}(v), \quad j=1,2
$$

where $I$ is the laser light intensity and $I_{\mathrm{S}}$ is the saturation intensity parameter. 
The peak value of $\sigma g$ is defined as: $\sigma_{0}=\frac{8 \pi k \mu^{2}}{\hbar \gamma}$ with $\mu$ indicating the electric dipole matrix element of the excited transition $(|2\rangle \rightarrow|3\rangle$ or $|1\rangle \rightarrow|3\rangle)$ and $k=\omega / \mathrm{c}$. $L_{i}(\nu)$ describes the Lorentzian line profile

$$
\begin{aligned}
& L_{j}(v)=\frac{(\gamma / 2)^{2}}{\left(\Delta_{j}-x\right)^{2}+(\gamma / 2)^{2}}, \\
& \quad j=1,2 \quad \Delta_{1}=0, \quad \Delta_{2}=\Delta_{\mathrm{hf}}, \quad x=k v .
\end{aligned}
$$

We assume that initially, at $I=0$, both population densities of the ground-states are equal and the excited state is not populated,

$$
n_{3}^{0}(v)=0 \quad \text { and } \quad n_{1}^{0}(v)=n_{2}^{0}(v)=n^{0}(v) .
$$

Here $n^{0}(v)=N G(v)$, with $G(v)=\frac{1}{u \sqrt{\pi}} \mathrm{e}^{-\left(\frac{v}{u}\right)^{2}} ; u$ is the mean thermal velocity defined by the mass $m$ of the $\mathrm{K}$ atom and the cell temperature $T: 1 / u^{2}=m /(2 k T)$.

In Eqs. (1)-(4), the terms in $\sigma_{j}$ describe the light in- duced (stimulated) emission and absorption, while the ones proportional to $\gamma g$ represent the spontaneous decay rate from the excited state. The last terms introduce the nonradiative population relaxation; in vacuum cell such process is caused by the collisions of atoms with the cell walls and by the finite interaction time determined by the beam dimensions and the atoms thermal velocity.

Typically, in vacuum cells, $\Gamma$ is significantly smaller than $\gamma \approx 6 \mathrm{MHz}$ for $\mathrm{K}(\Gamma \approx 100 \mathrm{KHz}$ for mean thermal velocity $u \approx 300 \mathrm{~m} / \mathrm{s}$ and beam diameter $d \approx 3 \mathrm{~mm}$ ). Therefore, the last term of Eq. (3) has only a minor contribution to the population of the excited state and can, thus, be neglected.

Equations (1)-(4) satisfy the rate balance: $\dot{n}_{1}+\dot{n}_{2}+$ $\dot{n}_{3}=0$. In steady state we have: $\dot{n}_{1}=\dot{n}_{2}=\dot{n}_{3}=0$ and the analytical solution of the rate equations in this regime gives the following expressions:

$$
\begin{aligned}
& n_{1}=2 n^{0} \frac{\left(2 \sigma_{2}+\frac{\gamma}{2}+\Gamma\right)\left(\sigma_{1}+\frac{\gamma}{2}+\frac{\Gamma}{2}\right)-\left(\sigma_{1}+\frac{\gamma}{2}\right)\left(\sigma_{2}+\frac{\gamma}{2}+\frac{\Gamma}{2}\right)}{\left(2 \sigma_{2}+\frac{\gamma}{2}+\Gamma\right)\left(2 \sigma_{1}+\frac{\gamma}{2}+\Gamma\right)-\left(\sigma_{1}+\frac{\gamma}{2}\right)\left(\sigma_{2}+\frac{\gamma}{2}\right)}, \\
& n_{2}=2 n^{0} \frac{\left(2 \sigma_{1}+\frac{\gamma}{2}+\Gamma\right)\left(\sigma_{2}+\frac{\gamma}{2}+\frac{\Gamma}{2}\right)-\left(\sigma_{2}+\frac{\gamma}{2}\right)\left(\sigma_{1}+\frac{\gamma}{2}+\frac{\Gamma}{2}\right)}{\left(2 \sigma_{2}+\frac{\gamma}{2}+\Gamma\right)\left(2 \sigma_{1}+\frac{\gamma}{2}+\Gamma\right)-\left(\sigma_{1}+\frac{\gamma}{2}\right)\left(\sigma_{2}+\frac{\gamma}{2}\right)} .
\end{aligned}
$$

In the case of cells with $\mathrm{K}$ and buffer gas, collisions between the two atomic species lead to a completely different system behavior: first of all, $\mathrm{K}$ atoms are forced to spend more time in the laser beam volume and, therefore, a significant reduction of the decay rate $\Gamma$ is observed; this creates an increased interaction time and a reduced atom wall collision rate. On the other hand, collisions with buffer gas result in an increased excited level decay rate $\gamma=\gamma_{\text {radiative }}+\gamma_{\text {dephasing }}$ determined by the spontaneous radiative decay and the dephasing rate due to the collision between $\mathrm{K}$ and buffer gas atoms. As a consequence of these processes broadening of the optical transition and decrease of the saturation parameter $I_{\mathrm{S}}$ take place [5]. A further improvement of the rate equation model for the case of buffer gas is currently under development taking into account the processes described above.

In the next section, based on the model for the vacuum cell, we analyze the very important influence of the velocity changing collisions (VCC) phenomenon introduced by the buffer gas. Without buffer gas VCC happens only after atom-wall collision and affects only the noninteracting with the radiation "fresh atoms" ready to enter the illuminated volume and described by the presence of $n^{0}$ in equations $(1-3,5,6)$. The buffer gas presence introduces more frequent VCC acting during the atomlight interaction time. The analysis allows to qualitatively compare evacuated and buffer gas cells in terms of VCC contribution apart from the factors described above.

\subsection{Theoretical comparison of pure evacuated and buffer-gas $K$ cell}

Figure $2 \mathrm{a}$ shows the plots of the steady state solution (Eqs. (5) and (6)) for a vacuum cell with parameters: $\Gamma=125 \mathrm{kHz}, \gamma=6 \mathrm{MHz}$ and saturation parameter $I / I_{\mathrm{s}}=1$. The laser radiation is considered monochromatic, centered on the $|2\rangle \rightarrow|3\rangle$ transition Doppler profile (interaction with $n_{2}(v=0)$, zero frequency detuning). The dips due to the OP clearly show that, in vacuum cells, the population of atoms interacting with the laser and responsible for the coherent resonances (point A in Fig. 2a) significantly decreases.

In the particular case of $K$, due to the overlapped Doppler profiles of the ground-state transitions, the laser light interacts at the same time with atoms populating level $|1\rangle$ belonging to different velocity classes $\left(n_{1}(v \neq 0)\right)$. As a result, hyperfine OP takes place in both directions, i.e. from $n_{1}$ to $n_{2}$ and vice versa. The atoms are optically pumped to the corresponding velocity class of the neighbor level creating a peak in the population profile. In the case of buffer gas cell (Figs. 2c,d), on the contrary, velocity changing collisions lead to a complete Maxwellisation of the Doppler profiles, while the total population, proportional to the areas under the $n_{1}$ and $n_{2}$ plots of Fig. 2a, is changed by the optical pumping process, as it is shown in Fig. 2c. The net difference between dips and peaks in the ground-state populations depends on the laser frequency detuning. By properly choosing the laser detuning, it is possible to achieve a complete compensation of the hyperfine OP, with an 


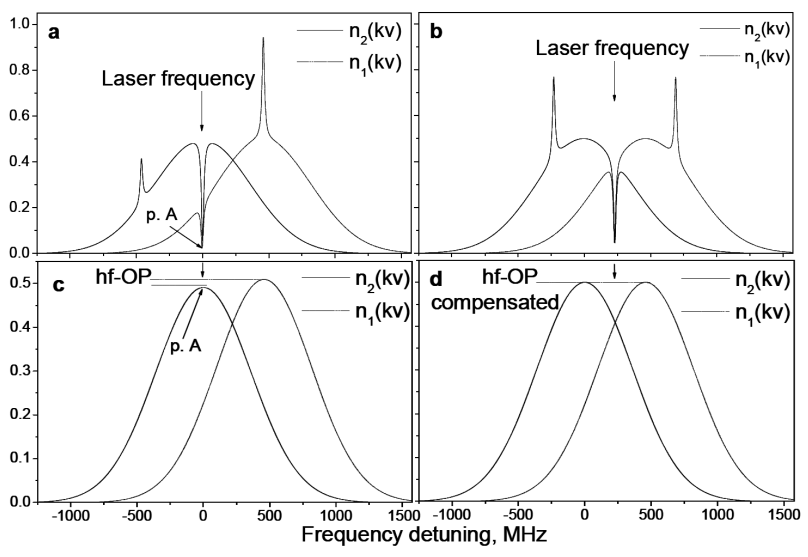

Fig. 2. Steady state solutions of Eqs. (1)-(4) describing $\mathrm{K}$ vacuum cell (a), (b). The corresponding plots in the rows below (c), (d) represent complete Maxwellisation induced by the collisions with buffer gas atoms of the calculated population distribution $n_{1}$ and $n_{2}$ illustrating the role of the velocity changing collisions.

equalization of the population distributions: comparison between Fig. 2b (evacuated cell) and 2d (cell with buffer gas) shows the active role of the VCCs. Such compensation is not possible for transitions with separated Doppler profiles.

In our study a simple three-level system is analyzed, which does not consider the Zeeman optical pumping. The illustration presented above is aimed just to stress on the influence of the profile overlapping on optical pumping. In case of completely separated transitions, the VCC do not contribute to optical pumping compensation.

\section{Experimental results}

In Fig. 3 we illustrate the contrast enhancement and the line width narrowing obtained in buffered $\mathrm{K}$ cell. Both cells were heated to the temperature of $55^{\circ} \mathrm{C}$ that corresponds to the maximum contrast of the EIT resonances obtained in those cells. Along with the typical difference in the resonances width of about two orders of magnitude, a significant enhancement of the resonance amplitude in absorption is clearly visible in the buffered cell (right): the resonance peak is about $6 \%$ for the vacuum cell while $35 \%$ for the buffer gas cell. Typically the buffer gas presence leads to reduction of the number of atoms participating on the EIT resonance preparation due to the atoms OP to a state no interacting with the light. Fig. 2 illustrates the particular for potassium OP compensation mechanism explaining the experimental comparison on Fig. 3. The situation for the cases of $\mathrm{Rb}$ and $\mathrm{Cs}$ is just the opposite resulting in bigger EIT resonance amplitude in the case of vacuum cell compared to buffer gas cell. We attribute that to the absence of hyperfine OP compensation as happens in $\mathrm{K}$.

We have pointed out an important advantage introduced by the particular property of potassium: partial
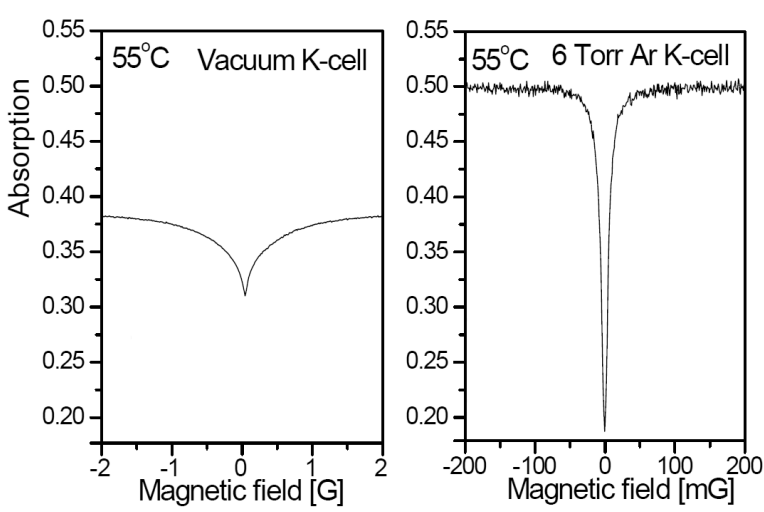

Fig. 3. Comparison between the EIT resonances obtained in Hanle configuration using a pure evacuated cell (left) and 6 Torr Ar cell (right) under the conditions described in [1].

or complete compensation of the hyperfine OP can be achieved in potassium cells filled with buffer gas, where velocity changing collisions take place. This is a result of the simultaneous excitation of atoms from both hyperfine levels and the VCC-involved velocity-groups Maxwellisation of the ground-state population. The hyperfine OP compensation is illustrated by just applying a Maxwellisation procedure on the $n_{1}(v)$ and $n_{2}(v)$ functions obtained for vacuum cell. A detailed description of the VCC dynamics in the differential equation system is under development. Experimental comparison of the amplitudes of EIT resonance obtained in vacuum and 6 Torr Ar-filled $\mathrm{K}$ cells is in qualitative agreement with the theoretical results.

\section{Acknowledgments}

This work was supported by the Italian National Research Council (bilateral cooperation program CNR/BAS and STM) and partially supported by the Bulgarian NCSR (grant No: DO 02-108/22.05.2009) and Indian-Bulgarian bilateral contract No. BIn-2/07.

\section{References}

[1] S. Gozzini, S. Cartaleva, A. Lucchesini, C. Marinelli, L. Marmugi, D. Slavov, T. Karaulanov, Eur. Phys. J. D 53, 153 (2009).

[2] S. Gozzini, S. Cartaleva, D. Slavov, L. Marmugi, A. Lucchesini, Proc. SPIE 7027, 70270K (2008).

[3] S. Pustelny, A. Wojciechowski, M. Gring, M. Kotyrba, J. Zachorowski, W. Gawlik, J. Appl. Phys. 103, 063108 (2008).

[4] F. de Tomasi, M. Allegrini, E. Arimondo, G.S. Agarwal, P. Ananthalakshmi, Phys. Rev. A 48, 5 (1993).

[5] P.G. Pappas, M.M. Burns, D.D. Hinshelwood, M.S. Feld, D.E. Murnick, Phys. Rev. A 21, 5 (1980). 